\title{
MicroRNA-532-5p regulates oxidative stress and insulin secretion damage in high glucose-induced pancreatic $\beta$ cells by downregulating the expression levels of CCND1
}

\author{
ZHIBIAO ZHONG ${ }^{1}$, WEILAN $\mathrm{SU}^{2}$ and HONGMEI CHEN ${ }^{3}$ \\ Departments of ${ }^{1}$ Occupational Diseases and ${ }^{2}$ Ultrasound, Shenzhen Prevention and Treatment Control Center for \\ Occupational Diseases, Shenzhen, Guangdong 518001; ${ }^{3}$ Department of Endocrinology and Metabolism, \\ The Second People's Hospital of Nantong, Nantong, Jiangsu 226000, P.R. China
}

Received August 3, 2020; Accepted November 20, 2020

DOI: $10.3892 / \mathrm{mmr} .2021 .12433$

\begin{abstract}
Diabetes mellitus is a metabolic disorder caused by insufficient insulin secretion. The expression of microRNA (miR)-532-5P is downregulated in diabetes, but its specific role in diabetes has not yet been elucidated. The present study aimed to investigate the specific mechanism underlying the effects of miR-532-5p on diabetes. Cell viability was determined using an MTT assay. The expression levels of miR-532-5P, cyclin D1 (CCND1), Insulin1 and Insulin2 were detected using reverse transcription-quantitative PCR. The expression of miR-532-5p and CCND1 were overexpressed in cells by cell transfection. ELISA was used to detect insulin secretion. 2',7'-dichlorodihydrofluorescein diacetate was used to quantify reactive oxygen species levels in cells. Apoptosis was detected using a TUNEL assay. Western blotting was performed to detect the expression of apoptosis-related proteins, CCND1 and $\mathrm{p} 53$. A dual-luciferase reporter assay was conducted, and verified the targeted binding of miR-532-5p and CCND1. The expression of miR-532-5p was downregulated in high glucose (HG)-induced MIN6 cells. Overexpression of miR-532-5p could improve the HG-induced decline in insulin secretion and inhibit HG-induced oxidative stress and apoptosis in cells. miR-532-5p can target and regulate the expression of CCND1. Overexpression of miR-532-5p downregulated HG-induced cell insulin secretion, oxidative stress and apoptosis by downregulating CCND1, which is involved in regulating the expression of $\mathrm{p} 53$. To conclude, miR-532-5p regulated oxidative stress and insulin secretion damage in HG-induced pancreatic $\beta$ cells by downregulating the expression of CCND1, which is involved in the upregulation of the expression of p53.
\end{abstract}

Correspondence to: Dr Hongmei Chen, Department of Endocrinology and Metabolism, The Second People's Hospital of Nantong, 298 Xinhua Road, Nantong, Jiangsu 226000, P.R. China E-mail: hongmeicc0@126.com

Key words: microRNA-532-5p, cyclin D1, diabetes, oxidative stress, insulin secretion damage

\section{Introduction}

Diabetes is a chronic disease involving metabolic disorders of sugar, protein and fat that is primarily caused by insufficient insulin in the body. $\beta$ cells are endocrine cells that secrete insulin. Insulin secretion by islet $\beta$ cells is mainly influenced by blood glucose levels $(1,2)$. A sharp decrease in the number of islet $\beta$ cells results in insufficient insulin secretion by cells, which in turn induces metabolic disorders of proteins, glucose and other substances. Among others, amino acids, hormones and hyperglycemia are all inducers of the abnormal function of islet $\beta$ cells, among which hyperglycemia is the most important one $(3,4)$. It is of great significance to investigate the underlying mechanism of damaged islet cells in a high glucose (HG) environment.

MicroRNAs (miRNAs/miRs) are an important regulator of numerous physiological and pathophysiological processes, and serve a key role in a number of biological processes, such as cell proliferation, differentiation, apoptosis and carcinogenesis (5). A previous study demonstrated that miRNAs promote insulin secretion and regulate insulin resistance by acting on multiple pathways, and abnormal miRNA expression may be the underlying pathogenesis of diabetes (6). It has been reported that miR-532-5p expression is downregulated in the plasma of patients with type 2 diabetes (7). miR-532-5p expression is downregulated in $\mathrm{H} 9 \mathrm{C} 2$ cells exposed to hypoxia, and in the myocardium of rats with acute myocardial infarction, reducing the apoptosis of $\mathrm{H} 9 \mathrm{C} 2$ cells induced by hypoxia (8). However, to the best of our knowledge, the specific role of miR-532-5p in diabetes has not been reported.

By querying the StarBase website, it was identified that miR-532-5p could target cyclin D1 (CCND1).CCND1, a member of the cyclin family, is a regulator of cyclin-dependent kinase. CCND1 expression has been reported to be upregulated in diabetic islets (9). Shen and Zhu (10) analyzed gene expression in type 2 diabetes and identified 124 upregulated differentially expressed genes, including CCND1, in the GSE15653 dataset, and these were associated with fatty acid and glucose metabolic pathways, and oxidation/reduction reactions, and may be involved in the development of obesity-related type 2 diabetes. In addition, in multiple myeloma, CCND1 can control the 
redox metabolism by producing reactive oxygen species (ROS) to disrupt redox balance (11). Furthermore, CCND1 silencing can damage the repair of DNA double-strand breaks, induce $\mathrm{G}_{0} / \mathrm{G}_{1}$ phase cell cycle arrest and inhibit ovarian cancer cell proliferation (12).

The apoptotic activation gene p53 induces cell cycle stagnation at the $\mathrm{G}_{0}$ stage, as well as apoptosis. A previous study demonstrated that $\mathrm{p} 53$ serves an important role in the initiation of apoptosis under different physiological conditions (13). Following treatment with $\mathrm{HG}$, the expression levels of p53 in INS-1 cells increased in a concentration-dependent manner, and overexpression of p53 induces apoptosis and reduces insulin secretion (14). Adaptive EGF expression sensitizes pancreatic cancer cells to ionizing radiation via activation of the CCND1/p53/poly(ADP-ribose) polymerase (PARP) signaling pathway (15). Therefore, it was speculated that miR-532-5p may regulate oxidative stress and insulin secretion damage of pancreatic $\beta$ cells induced by HG by regulating $\mathrm{CCND1} / \mathrm{p} 53$.

The present study examined the regulatory effects of miR-532-5p on diabetes and explored the underlying mechanisms by inducing islet cells with HG. The present study provided a theoretical basis for the investigation of the underlying mechanisms of diabetes and potential drug targets.

\section{Materials and methods}

Cell lines and culture conditions. The $\beta$ cell line of the pancreas (MIN6) was obtained from The Cell Bank of Type Culture Collection of the Chinese Academy of Sciences and incubated in DMEM (Gibco; Thermo Fisher Scientific, Inc.) supplemented with 10\% FBS (Gibco; Thermo Fisher Scientific, Inc.) at $37^{\circ} \mathrm{C}$ with $5 \% \mathrm{CO}_{2}$. In the present experiment, the normal glucose control (5 $\mathrm{mM}$ glucose, $\mathrm{NG}$ group) was set as a control group, the mannitol group (MA) was set up to exclude the osmotic pressure effects of HG on cells. The MIN6 cells were incubated in complete medium containing $25 \mathrm{mM}$ glucose (final concentration in the medium) for $24 \mathrm{~h}$, which was called the HG group (16). The HG + mimic-NC group was transfected with a mimic-NC and then induced with $25 \mathrm{mM}$ glucose. The HG + miR-532-5p mimic group was transfected with the miR-532-5p mimic and then induced with $25 \mathrm{mM}$ glucose. The HG + miR-532-5p mimic + pcDNA-NC group were transfected with miR-532-5p mimic and the pcDNA-NC plasmid, and were then induced with $25 \mathrm{mM}$ glucose. The HG + miR-532-5p mimic + pcDNA-CCND1 group was transfected with miR-532-5p mimic and the pcDNA-CCND plasmid, and were then induced with $25 \mathrm{mM}$ glucose.

Database selection and analysis. StarBase (http://starbase. sysu.edu.cn/) was used to identify the binding sites of miR-532-5p and CCND1.

MTT assay. Cells were seeded at a density of $1 \times 10^{4}$ cells/well in 96-well plates. Following treatment of the cells, $20 \mu \mathrm{l}$ MTT solution $(5 \mathrm{mg} / \mathrm{ml}$; Gen-view Scientific, Inc.) was added to each well and cells were incubated at $37^{\circ} \mathrm{C}$ with $5 \% \mathrm{CO}_{2}$ for $4 \mathrm{~h}$. Subsequently, $100 \mu \mathrm{l}$ DMSO was added to dissolve the formazan crystals at $37^{\circ} \mathrm{C}$ for $10 \mathrm{~min}$. The optical density at $490 \mathrm{~nm}$ was measured the following day to determine the quantities of formazan formed by cleaving of MTT in living cells.

Reverse transcription-quantitative PCR (RT-qPCR). Total RNA was extracted from cells using TRIzol ${ }^{\circledR}$ reagent (Invitrogen; Thermo Fisher Scientific, Inc.) and then reverse transcribed into cDNA using the First-Strand cDNA Synthesis kit (Invitrogen; Thermo Fisher Scientific, Inc.). The SuperScript ${ }^{\mathrm{TM}}$ III Platinum ${ }^{\mathrm{TM}}$ SYBR Green One-Step qRT-PCR kit (Invitrogen; Thermo Fisher Scientific, Inc.) was used according to the manufacturer's protocols as follows: $95^{\circ} \mathrm{C}$ for $10 \mathrm{~min}, 40$ cycles of $95^{\circ} \mathrm{C}$ for $10 \mathrm{sec}, 55^{\circ} \mathrm{C}$ for $10 \mathrm{sec}$ and $72^{\circ} \mathrm{C}$ for $30 \mathrm{sec}$. Relative expression levels were calculated according to the $2^{-\Delta \Delta \mathrm{Cq}}$ method (17). The following primers were used: miR-532-5p forward, 5'-GCGCGCATGCCTTGAGTG TAG-3' and reverse, 5'-ATCCAGTGCAGGGTCCGAGG-3'; CCND1 forward, 5'-ACGAAGGTCTGCGCGTGTT-3' and reverse, 5'-CCGCTGGCCATGAACTACCT-3'; p53 forward, 5'-GGCCCACTTCACCGTACTAA-3' and reverse, 5'-GTG GTTTCAAGGCCAGATGT-3'; U6 forward, 5'-CTCGCT TCGGCAGCACA-3' and reverse, 5'-AACGCTTCACGAATT TGCGT-3'; Insulin1 forward, 5'-TAGTGACCAGCTATAATC AGAG-3' and reverse, 5'-ACGCCAAGGTCTGAAGGTCC-3'; Insulin2 forward, 5'-CCCTGCTGGCCCTGCTCTT3-3' and reverse, 5'-AGGTCTGAAGGTCACCTGCT-3'; and GAPDH forward, 5'-ACCACAGTCCATGCCATCAC-3' and reverse, 5'-TCCACCACCCTGTTGCTGTA-3'. Insulin1 and Insulin2 were detected as transcripts of the insulin gene, which are markers of pancreatic $\beta$ cells (18). GAPDH was used as the housekeeping gene for CCND1, Insulin1, Insulin2 and p53. U6 was used as the housekeeping gene for miR-532-5p.

Cell transfection. Cells $\left(1 \times 10^{5}\right.$ cells/well $)$ were seeded into 6-well plates and cultured for $24 \mathrm{~h}$ at $37^{\circ} \mathrm{C}$ with $5 \% \mathrm{CO}_{2}$. Cell transfection was performed when the cells reached $80 \%$ confluency. The miR-532-5p mimic and mimic-negative control (mimic-NC; Invitrogen; Thermo Fisher Scientific, Inc.) were transfected directly into cells. For overexpression of miR-532-5p, the cells were transfected with mimic at a final concentration of $25 \mathrm{nM}$ for $48 \mathrm{~h}$ at $37^{\circ} \mathrm{C}$. For pcDNA-NC (empty vector, Invitrogen; Thermo Fisher Scientific, Inc.) and pcDNA-CCND1, full length transcript of CCND1 was amplified from cDNA obtained from 293T cells by PCR using PrimeSTAR ${ }^{\circledR}$ HS DNA polymerase (Takara Bio, Inc.), and was transfected at a final concentration of $500 \mathrm{ng}$ for $48 \mathrm{~h}$ at $37^{\circ} \mathrm{C}$. The PCR amplification product was inserted into the KpnI and BamHI sites of the pcDNA vector (Invitrogen; Thermo Fisher Scientific, Inc.), which was termed pcDNA-CCND1. Transfection was performed using Lipofectamine ${ }^{\circledR} 2000$ (Invitrogen; Thermo Fisher Scientific, Inc.) according to the manufacturer's protocol. Transfection efficiency was determined by RT-qPCR $48 \mathrm{~h}$ after transfection.

ELISA. Cytokine concentration normalized to total insulin was detected using ELISA kits (ELISA MAX ${ }^{\mathrm{TM}}$ Deluxe Set Human IGFALS; cat. no. 445904 BioLegend, Inc.) (16). After the cells were centrifuged $\left(300 \mathrm{xg}, 10 \mathrm{~min}, 37^{\circ} \mathrm{C}\right)$, cell-free supernatant was diluted to $100 \mu \mathrm{l}(1: 20)$ and incubated with the specific capture antibody and detection antibody. Measurements were made at an optical density of $450 \mathrm{~nm}$. 
ROS assay. ROS levels of cells were detected using the fluorescent probe 2',7'-dichlorodihydrofluorescein diacetate (Sigma-Aldrich; Merck KGaA), which could be rapidly oxidized into the fluorescent 2',7'-dichlorofluorescein (DCF) in the presence of intracellular ROS. Fluorescence was monitored with a laser scanning confocal microscope (Leica Microsystems $\mathrm{GmbH}$ ) at $488 \mathrm{~nm}$ (magnification, x200). The amount of ROS was quantified as the relative fluorescence intensity of DCF per cell in the scanned area.

TUNEL assay. A total of $3 \times 10^{4}$ cells were seeded in 24-well plates and incubated overnight. The cells were treated after fusion. Cells were fixed with $4 \%$ paraformaldehyde for $30 \mathrm{~min}$ at room temperature and then washed with PBS. Subsequently, $0.3 \%$ Triton X-100 in PBS was added and incubated for $5 \mathrm{~min}$. The cells were collected and washed with PBS three times, and treated with $50 \mu 1$ TUNEL assay solution (Roche Diagnostics $\mathrm{GmbH}$ ) at $37^{\circ} \mathrm{C}$ in the dark for $60 \mathrm{~min}$, followed by the addition of stop solution. Subsequently, cells were incubated with DAB solution and stained with hematoxylin and eosin for $5 \mathrm{~min}$ at room temperature according to the manufacturer's protocol. Stained apoptotic cells were visualized at x 20 magnification under an LSM 710 laser scanning confocal microscope (Carl Zeiss AG).

Western blotting. Cells were collected, lysed with RIPA lysis buffer (Beyotime Institute of Biotechnology) and incubated for $30 \mathrm{~min}$ on ice. Subsequently, proteins were detected using a BCA protein assay kit (Bio-Rad Laboratories, Inc.). A total of $40 \mu \mathrm{g}$ protein was loaded onto $10 \%$ SDS-polyacrylamide gels to separate proteins, which were subsequently transferred to PVDF membranes. The membranes were blocked with $10 \%$ skimmed milk for $2 \mathrm{~h}$ at room temperature, followed by incubation overnight at $4^{\circ} \mathrm{C}$ with the following primary antibodies: Anti-Bax (1:1,000; cat. no. 14796S; Cell Signaling Technology, Inc.), anti-caspase-3 (1:1,000; cat. no. 700182; Thermo Fisher Scientific, Inc.), anti-cleaved caspase-3 (1:1,000; cat. no. PA5-17913; Thermo Fisher Scientific, Inc.), anti-Bcl-2 (1:1,000; cat. no. 15071S; Cell Signaling Technology, Inc.), anti-CCND1 (1:1,000; cat. no. MA5-14512; Thermo Fisher Scientific, Inc.), anti-P53 (1:1,000; cat. no. MA5-12557; Thermo Fisher Scientific, Inc.) and anti-GAPDH (1:1,000; cat. no. 5174S; Cell Signaling Technology, Inc.). Subsequently, the membranes were incubated with goat anti-rabbit horseradish peroxidase-conjugated IgG secondary antibodies $(1: 5,000$; cat. nos. A32731 and A11032; Thermo Fisher Scientific, Inc.) at room temperature for $1 \mathrm{~h}$. The signals were detected using enhanced chemiluminescence reagent (GE Healthcare), and ImageJ software (version 146; National Institutes of Health) was used to analyze the fold changes of protein levels.

Luciferase reporter assays. To validate the direct targeting of miR-532-5p, the $3^{\prime}$ untranslated region (3'UTR) of the putative target gene CCND1 was cloned into the psiCHECK2 vector (Promega Corporation), according to the manufacturer's instructions. The mutated or wild-type CCND1 cells were divided into mimic-NC + CCND1 group and miR-532-5p mimic + CCND1 group. Vectors containing the respective 3'UTRs were co-transfected with miRNA mimic (5'-CAUGCC UUGAGUGUAGGACCGU-3') into cells ( $1 \times 10^{6}$ cells) at a final
A

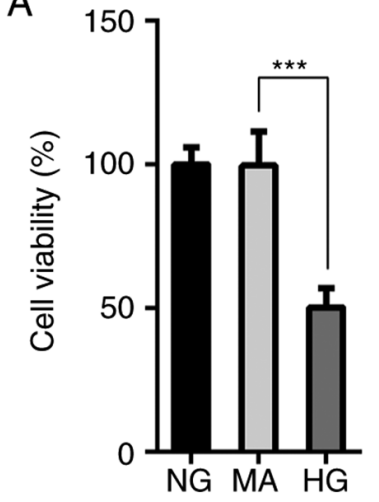

B

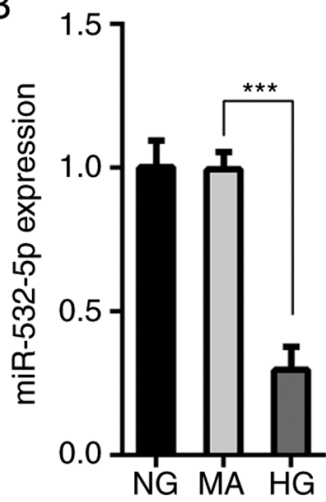

Figure 1. Cell viability and miR-532-5p expression in HG-induced cells. (A) Cell Counting Kit-8 assay was performed to detect cell viability. (B) Reverse transcription-quantitative PCR was used to detect the expression of miR-532-5p. ${ }^{* * * *} \mathrm{P}<0.001$. HG, high glucose; miR, microRNA; MA, mannitol group; $\mathrm{NG}$, normal glucose.

concentration of $500 \mathrm{ng}$ for $48 \mathrm{~h}$ at $37^{\circ} \mathrm{C}$ using Lipofectamine 2000 according to the manufacturer's protocol. Mutations in each of the predicted target sites in CCND1 3'UTRs were generated by site-directed mutagenesis using the QuikChange II Site-Directed Mutagenesis kit (Agilent Technologies, Inc.) according to the manufacturer's protocols. Subsequently, the cells were washed with PBS and lysed with cell lysis buffer (Beyotime Institute of Biotechnology) after transfection. The luciferase activity was measured using a plate reader (BD Biosciences) and was normalized to Renilla luciferase activity (pRL-TK) using the Luc-Screen ${ }^{\mathrm{TM}}$ Extended-Glow Luciferase Reporter Gene Assay system (cat. no. T1033; Thermo Fisher Scientific, Inc.). All procedures were conducted according to the manufacturers' instructions. Luciferase gene plasmid (containing WT and Mut UTRs) was constructed by Thermo Fisher Scientific, Inc.

Statistical analysis. Data are presented as the mean \pm standard deviation. Each experiment was repeated three times. SPSS version 19.0 software (IBM Corp.) was used to perform statistical analysis. Comparisons among multiple groups were analyzed using one-way ANOVA followed by Tukey's post hoc test. $\mathrm{P}<0.05$ was considered to indicate a statistically significant difference.

\section{Results}

Overexpression of miR-532-5p improves the impaired functions of secreted insulin in HG-induced cells. The MTT assay results demonstrated that compared with that of cells in the NG and MA groups, the survival rate of cells in the HG group was decreased (Fig. 1A), and this was accompanied by a decrease in the expression levels of miR-532-5p (Fig. 1B). This suggested that miR-532-5p served an important role in HG-induced cells. Subsequently, miR-532-5p was overexpressed using the cell transfection technique, and transfection efficiency was measured by RT-qPCR (Fig. 2A). Furthermore, cells were divided into NG, MA, $\mathrm{HG}, \mathrm{HG}+$ mimic-NC and $\mathrm{HG}+$ miR-532-5p mimic groups. ELISA was used to detect the functions of secreted insulin. 

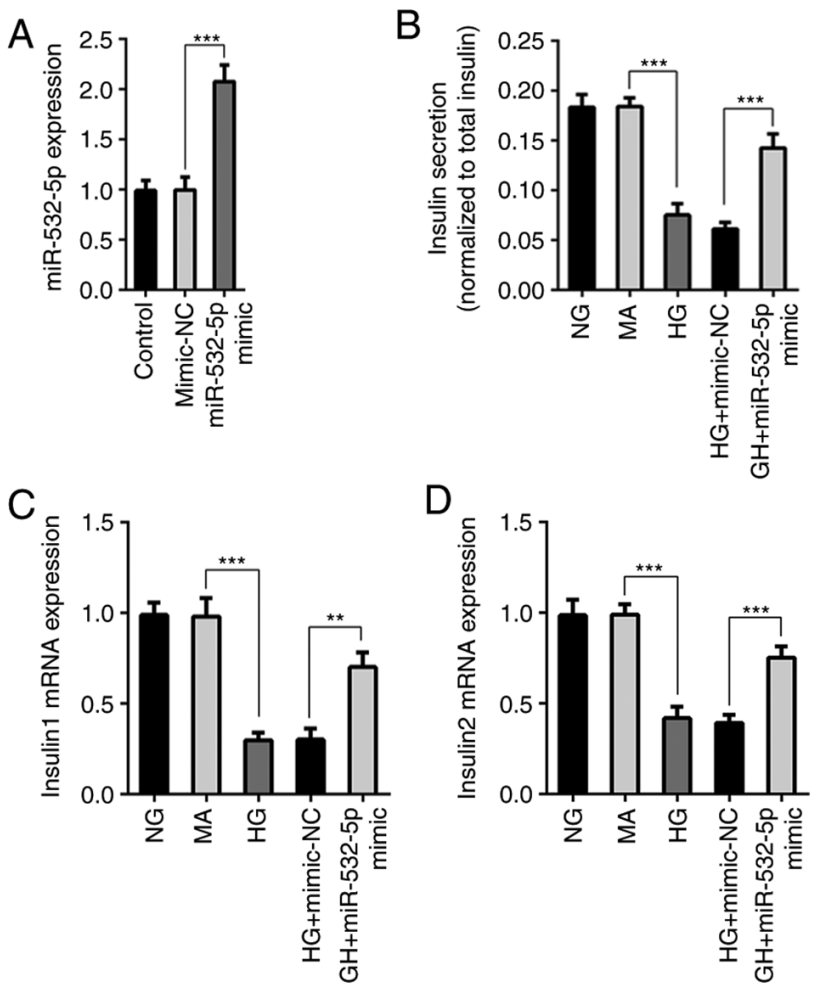

Figure 2. Overexpression of miR-532-5p induces insulin secretion in HG-induced cells. (A) RT-qPCR was performed to detect the expression of miR-532-5p following transfection with miR mimic and NC. (B) ELISA assay was conducted to detect the levels of insulin secretion. The expression levels of (C) Insulin1 and (D) Insulin2 were detected using RT-qPCR ${ }^{* * *} \mathrm{P}<0.01,{ }^{* * *} \mathrm{P}<0.001$. HG, high glucose; miR, microRNA; MA, mannitol group; NG, normal glucose; RT-qPCR, reverse transcription-quantitative PCR; NC, negative control.

Compared with those in the NG and MA groups, the insulin secretion levels in the HG group were decreased. Compared with those of cells in the $\mathrm{HG}+$ mimic-NC group, the insulin secretion levels of cells in the HG + miR-532-5p mimic group were increased (Fig. 2B). In addition, RT-qPCR was used to detect the gene transcription levels of Insulin1 and Insulin2, and the trend was consistent with that of the total insulin level (Fig. 2C and D). The results demonstrated that overexpression of miR-532-5p improved the impaired functions of secreted insulin in HG-induced cells.

Overexpression of miR-532-5p inhibits oxidative stress levels in HG-induced cells. Subsequently, the levels of ROS were detected. Compared with those in the NG and MA groups, the ROS levels in the HG group were increased. Additionally, following overexpression of miR-532-5p, the ROS levels were decreased compared with the $\mathrm{HG}+$ mimic-NC group (Fig. 3A and B). TUNEL staining was subsequently used to detect apoptosis. Compared with the NG and MA groups, the HG group exhibited an increased apoptosis rate (Fig. 4A), and this was accompanied by increased expression levels of Bax and cleaved caspase-3, and decreased expression levels of Bcl-2 (Fig. 4B). Additionally, compared with that of cells in the $\mathrm{HG}+$ mimic-NC group, the apoptosis rate of cells in the $\mathrm{HG}+$ miR-532-5p mimic group exhibited a significant decline, and this was accompanied by decreased expression levels of Bax and cleaved caspase-3, and increased expression levels of
Bcl-2. The results revealed that overexpression of miR-532-5p could inhibit oxidative stress levels in HG-induced cells.

Overexpression of miR-532-5p downregulates CCNDI expression in $H G$-induced cells. StarBase was used to identify that miR-532-5p could target the 3'UTR of CCND1 (Fig. 5A). Additionally, a luciferase reporter assay was used to verify the targeted binding of miR-532-5p and CCND1. The results demonstrated that before CCND1 mutation, luciferase activity was significantly decreased in the miR-532-5p mimic + CCND1 group compared with that in the Vector + CCND1 group. After CCND1 mutation, the luciferase activity was not significantly altered between the Vector + CCND1 and miR-532-5p mimic + CCND1 groups (Fig. 5B). Expression levels of CCND1 in cells were detected following overexpression of miR-532-5p. As shown in Fig. 5C and D, compared with those in the NG and MA groups, the expression levels of CCND1 in the HG group were significantly increased. Compared with those in the $\mathrm{HG}+$ mimic-NC group, the expression levels of CCND1 in the HG + miR-532-5p mimic group were decreased.

Overexpression of miR-532-5p improves the impaired functions of secreted insulin and inhibits oxidative stress levels in HG-induced cells by downregulating CCND1 expression. Compared with those in the pcDNA-NC group, the expression levels of CCND1 in the pcDNA-CCND1 group were significantly increased, indicating successful overexpression (Fig. 6A and B). Subsequently, the cells were divided into $\mathrm{NG}, \mathrm{HG}, \mathrm{HG}+$ mimic-NC, $\mathrm{HG}+\operatorname{miR}-532-5 \mathrm{p}$ mimic, $\mathrm{HG}+\operatorname{miR}-532-5 \mathrm{p}$ mimic + pcDNA-NC and $\mathrm{HG}+\operatorname{miR}-532-5 \mathrm{p}$ mimic + PCDNA-CCND1 groups. The secretion of insulin by the cells was detected using ELISA. Compared with those in the $\mathrm{HG}+$ miR-532-5p mimic + pcDNA-NC group, the total insulin levels in the $\mathrm{HG}+\operatorname{miR}-532-5 \mathrm{p}$ mimic + pcDNA-CCND1 group were decreased (Fig. 6C), as were the levels of Insulin1 (Fig. 6D) and Insulin2 (Fig. 6E). Subsequently, ROS levels were detected, and the trend was the opposite of that detected for insulin (Fig. 7A and B). Apoptosis was detected using a TUNEL assay. Compared with that in the $\mathrm{HG}+$ miR-532-5p mimic + pcDNA-NC group, apoptosis in the $\mathrm{HG}+\operatorname{miR}-532-5 \mathrm{p}$ mimic + pcDNA-CCND1 group increased (Fig. 7C and D), and this was accompanied by increased expression levels of Bax and cleaved caspase-3, and decreased expression levels of Bcl-2 (Fig. 7E). These results indicated that overexpression of miR-532-5p improved the impaired functions of secreted insulin and inhibited oxidative stress levels in HG-induced cells by downregulating CCND1 expression.

Overexpression of $m i R-532-5 p$ regulates the expression levels of p53 in HG-induced cells by downregulating CCNDI expression. During the study, abnormal expression levels of p53 were observed in the cells (Fig. 8A and B). Compared with those in the NG group, the expression levels of p53 in the cells were increased following HG induction, whereas the expression levels of p53 in the cells were decreased following overexpression of miR-532-5p. Compared with that in the $\mathrm{HG}+$ miR-532-5p mimic + pcDNA-NC group, p53 expression in the $\mathrm{HG}+\operatorname{miR}-532-5 \mathrm{p}$ mimic + pcDNA-CCND1 group was increased. Therefore, it was preliminarily concluded that overexpression of $\mathrm{miR}-532-5 \mathrm{p}$ regulated the expression 
A

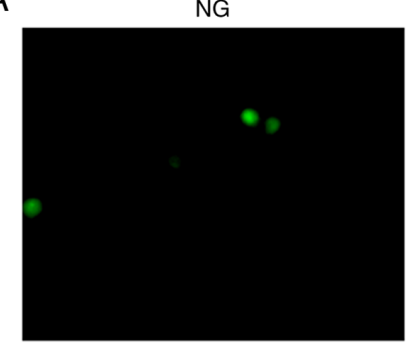

$\mathrm{HG}+$ mimic-NC

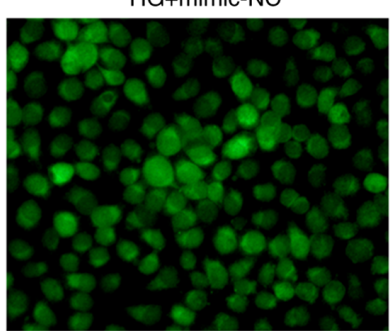

MA

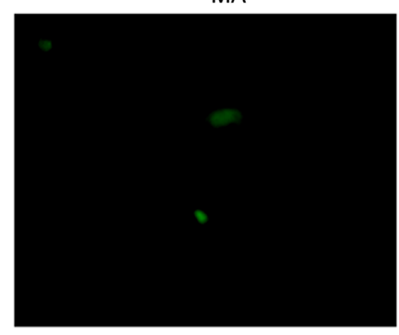

$H G+m i R-532-5 p$ mimic

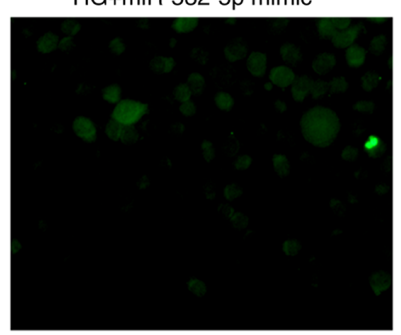

$\mathrm{HG}$

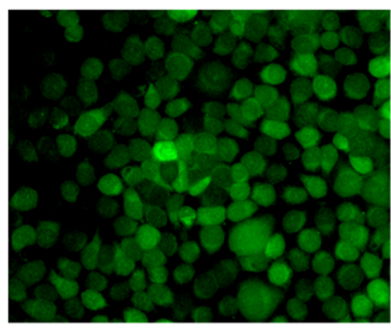

B

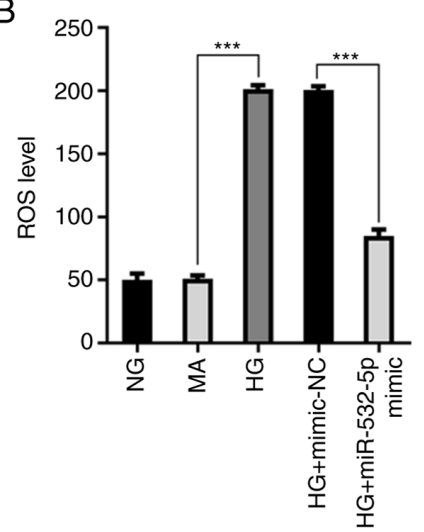

Figure 3. Overexpression of miR-532-5p inhibits oxidative stress levels in HG-induced cells. (A) 2',7'-dichlorodihydrofluorescein diacetate fluorescence probe was used to detect ROS expression. (B) Quantification of ROS expression. ${ }^{* * *} \mathrm{P}<0.001$. HG, high glucose; miR, microRNA; MA, mannitol group; NG, normal glucose; NC, negative control; ROS, reactive oxygen species.

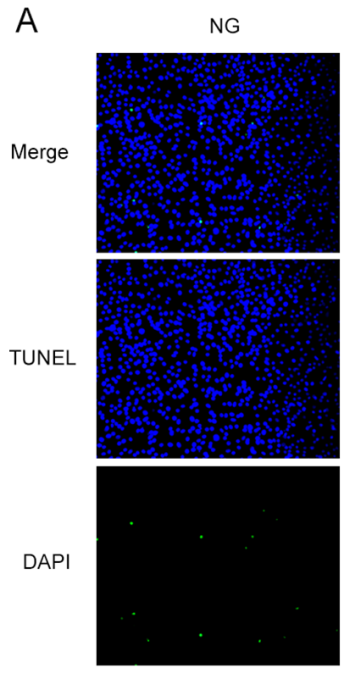

B

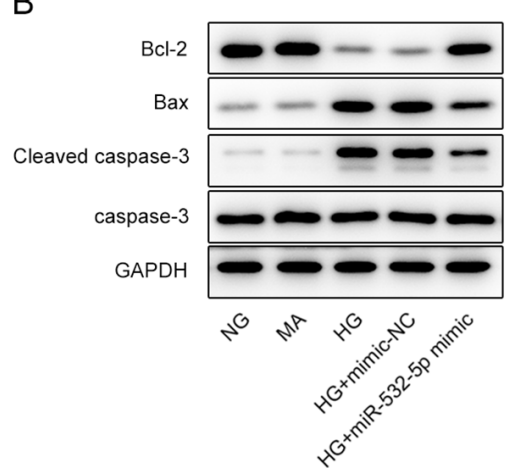

MA
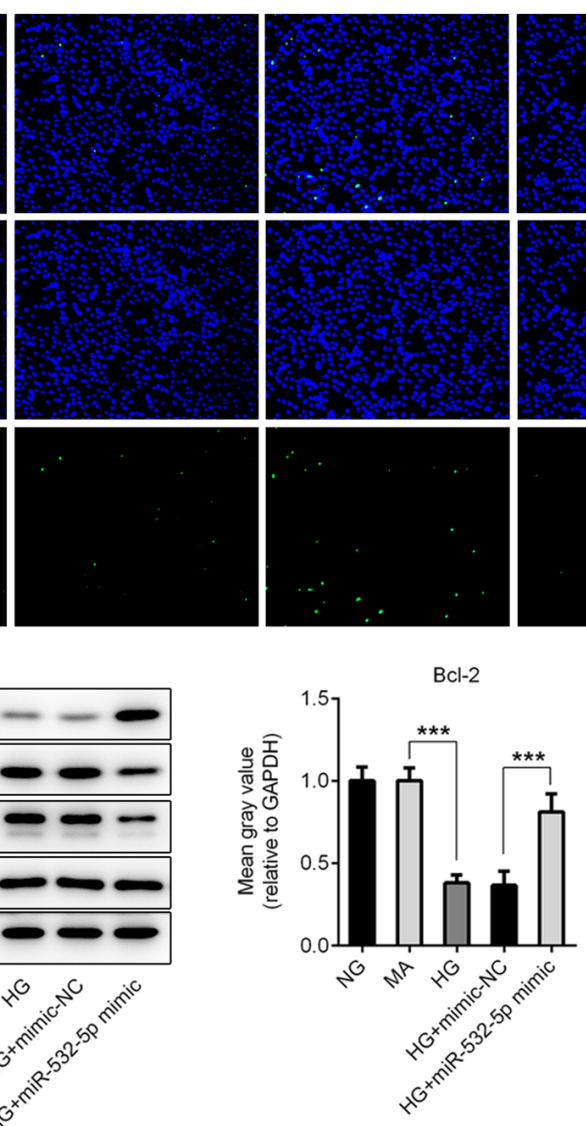

$\mathrm{HG}+$ mimic-NC
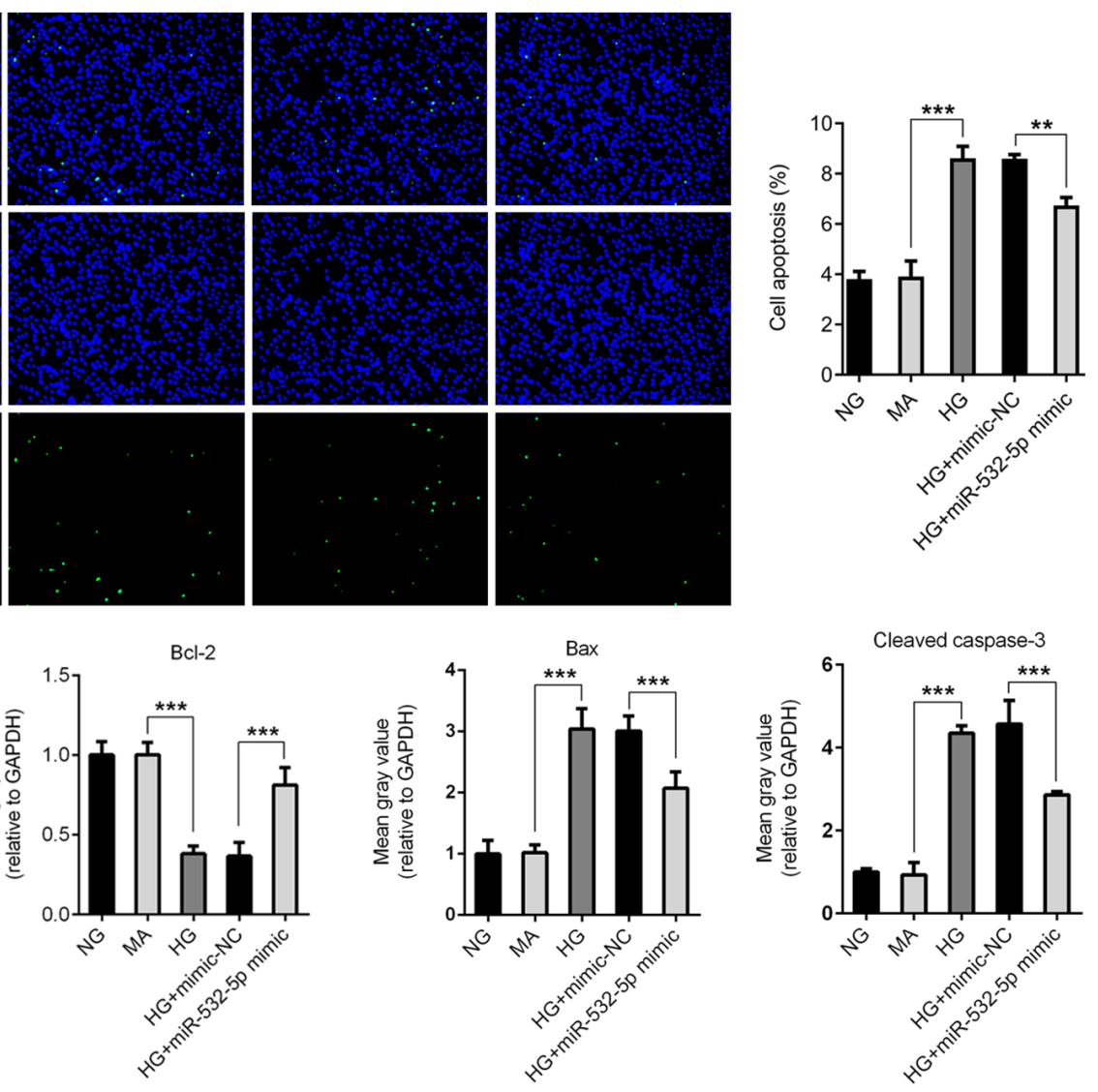

Figure 4. Overexpression of miR-532-5p inhibits apoptosis levels in HG-induced cells. (A) TUNEL assay was performed to detect the apoptosis rates of cells. (B) The expression levels of apoptosis-related proteins were detected using western blotting. ${ }^{* *} \mathrm{P}<0.01,{ }^{* * *} \mathrm{P}<0.001$. HG, high glucose; miR, microRNA; MA, mannitol group; NG, normal glucose; NC, negative control; ROS, reactive oxygen species. 
A CCND1 : 5' cuguagugggguucuAGGCAUc 3' IIIIII miR-532-5p: $3^{\prime}$ ugccaggaugugaguUCCGUAc 5'
D

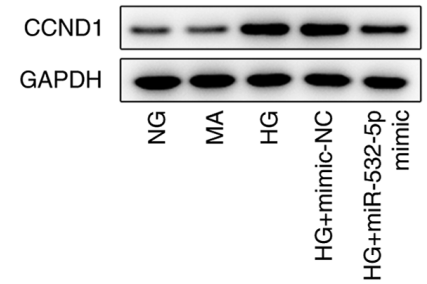

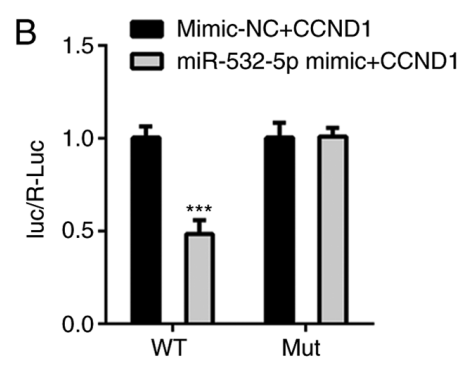

C

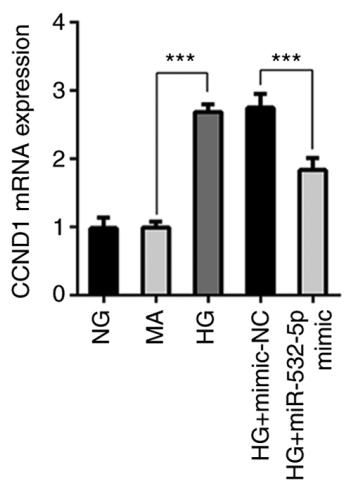

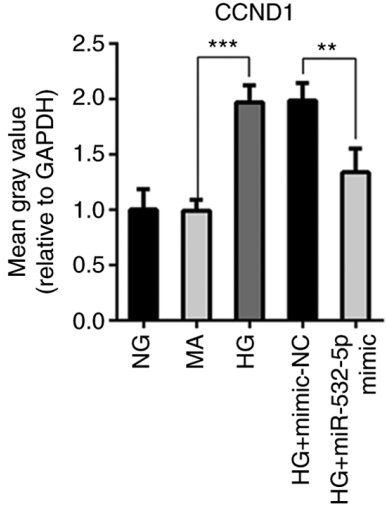

Figure 5. Overexpression of miR-532-5p downregulates CCND1 expression in HG-induced cells. (A) Targeted binding sites of miR-532-5p and CCND1. (B) Luciferase reporter gene assay was performed to verify the binding between miR-532-5p and CCND1. The expression of CCND1 was determined using (C) reverse transcription-quantitative PCR and (D) western blotting. ${ }^{* *} \mathrm{P}<0.01,{ }^{* * *} \mathrm{P}<0.001$. HG, high glucose; miR, microRNA; MA, mannitol group; NG, normal glucose; NC, negative control; CCND1, cyclin D1; WT, wild-type; Mut, mutant.

A

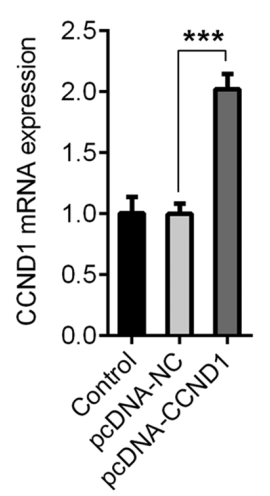

C

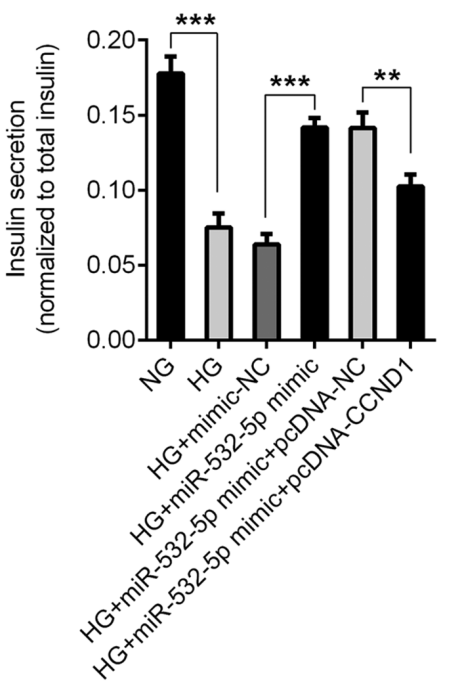

B
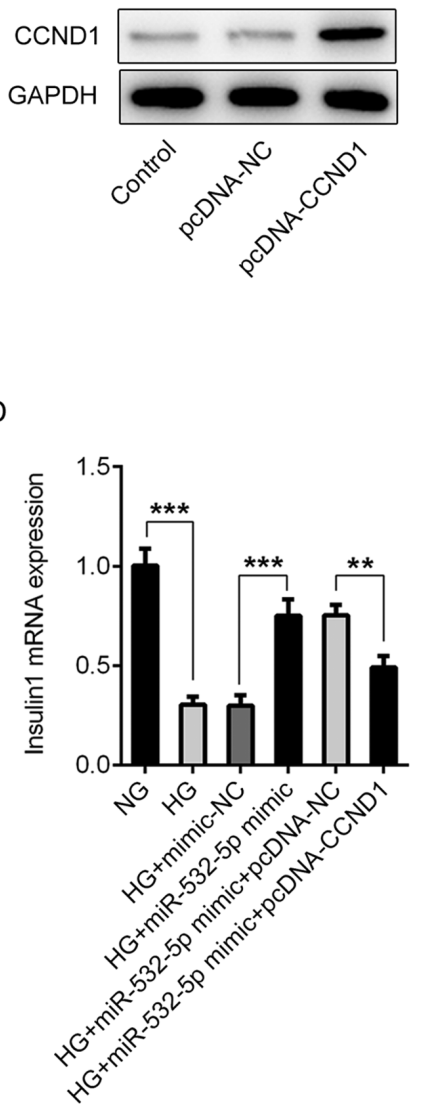

CCND1

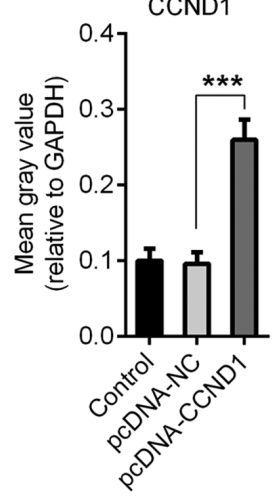

$\mathrm{E}$

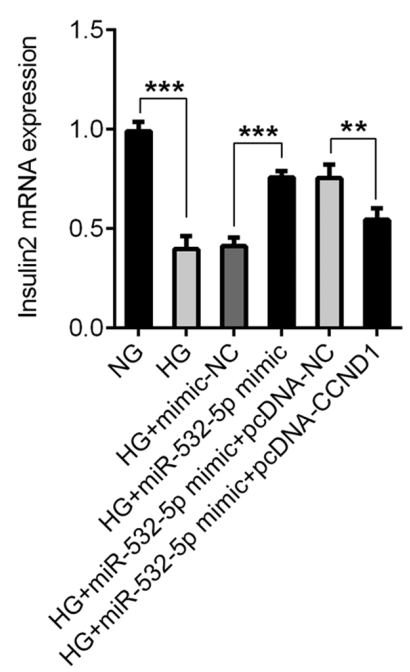

Figure 6. Overexpression of miR-532-5p improves the impaired functions of secreted insulin in HG-induced cells by downregulating CCND1. (A) RT-qPCR and (B) western blotting were performed to detect the expression of CCND1. (C) ELISA assay was used to detect the levels of insulin secretion. RT-qPCR was conducted to detect the expression levels of (D) Insulin1 and (E) Insulin2. ${ }^{* *} \mathrm{P}<0.01,{ }^{* * *} \mathrm{P}<0.001$. HG, high glucose; miR, microRNA; MA, mannitol group; NG, normal glucose; NC, negative control; CCND1, cyclin D1; RT-qPCR, reverse transcription-quantitative PCR. 
A

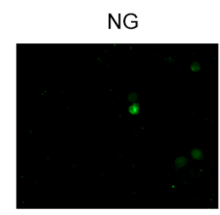

HG

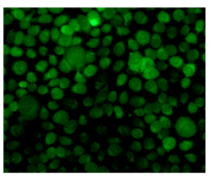

HG+mimic-NC
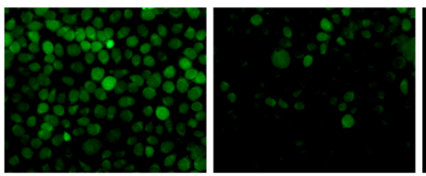

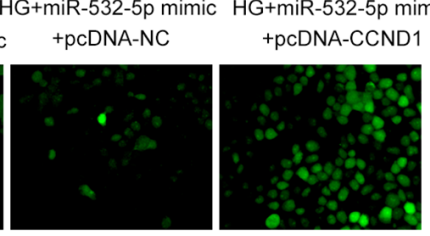

$H G+m i R-532-5 p$ mimic $H G+m i R-532-5 p$ mimic
B

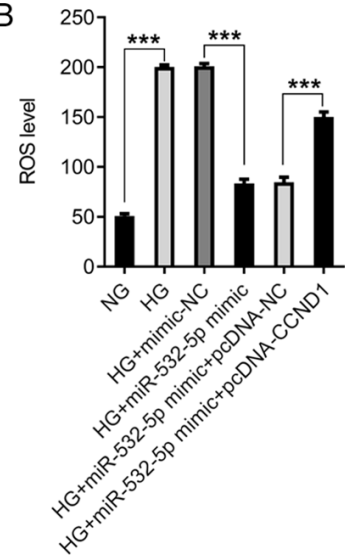

C
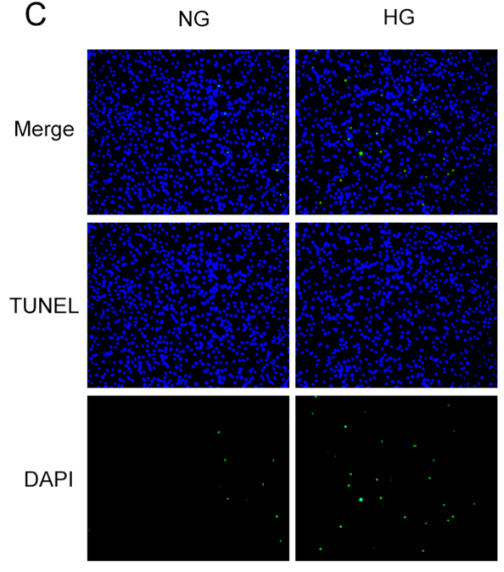

HG
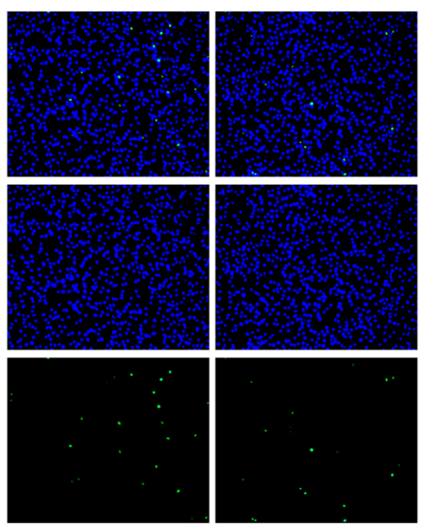
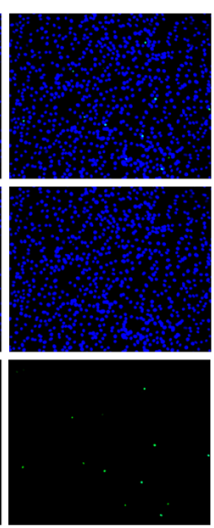

+miR-532-5p mim + pcDNA-CCND1
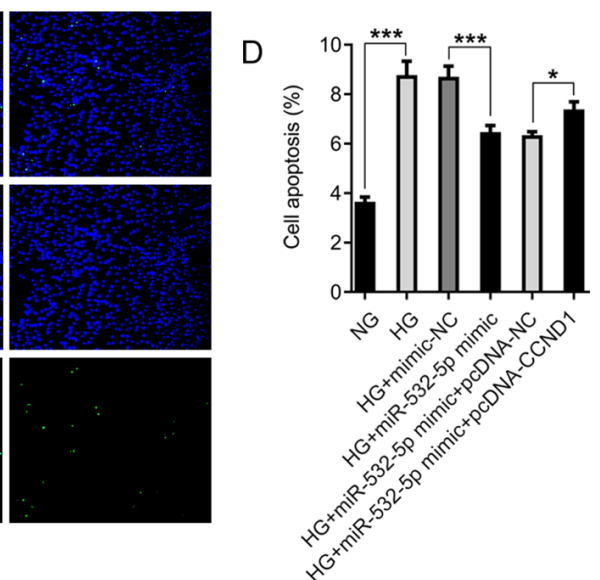

E

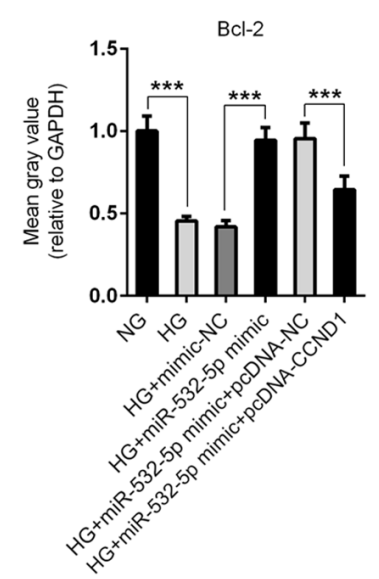

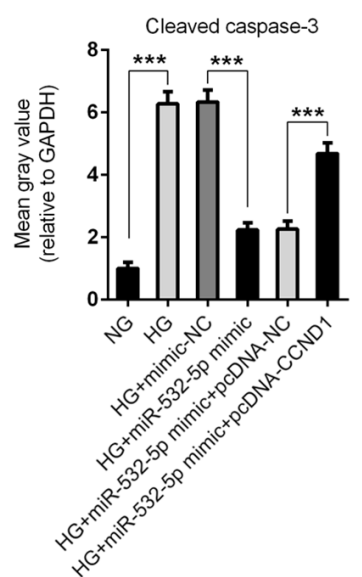

Figure 7. Overexpression of miR-532-5p inhibits oxidative stress levels in HG-induced cells by downregulating CCND1. (A) 2',7'-dichlorodihydrofluorescein diacetate fluorescence probe was used to detect ROS expression. (B) Quantification of ROS expression. (C) TUNEL assay was performed to detect the apoptosis rate of cells. (D) Quantification of apoptosis rates. (E) The expression levels of apoptosis-related proteins were determined using western blotting. ${ }^{*} \mathrm{P}<0.05,{ }^{* *} \mathrm{P}<0.01,{ }^{* * *} \mathrm{P}<0.001$. HG, high glucose; miR, microRNA; MA, mannitol group; NG, normal glucose; NC, negative control; CCND1, cyclin D1; ROS, reactive oxygen species.

levels of p53 in HG-induced cells by downregulating CCND1 expression.

\section{Discussion}

miRNAs have been considered as potential biomarkers of tissue-specific origin, which affect the occurrence and development of diabetes by participating in processes such as collective oxidative stress and the inflammatory response (19). miR-21-5p expression in extracellular vesicles is increased during inflammatory responses and serves as a biomarker for type 1 diabetes (20). Overexpression of miR-22 can reduce oxidative stress injury in diabetic cardiomyopathy via sirtuin 1 (21). miR-365 promotes diabetic retinopathy by inhibiting TIMP metallopeptidase inhibitor 3 and increasing oxidative stress (22). It has been reported that miR-532-5p expression is downregulated in patients with type 2 diabetes (6). In the present study, the expression levels of miR-532-5p were also markedly decreased in HG-induced MIN6 cells. 

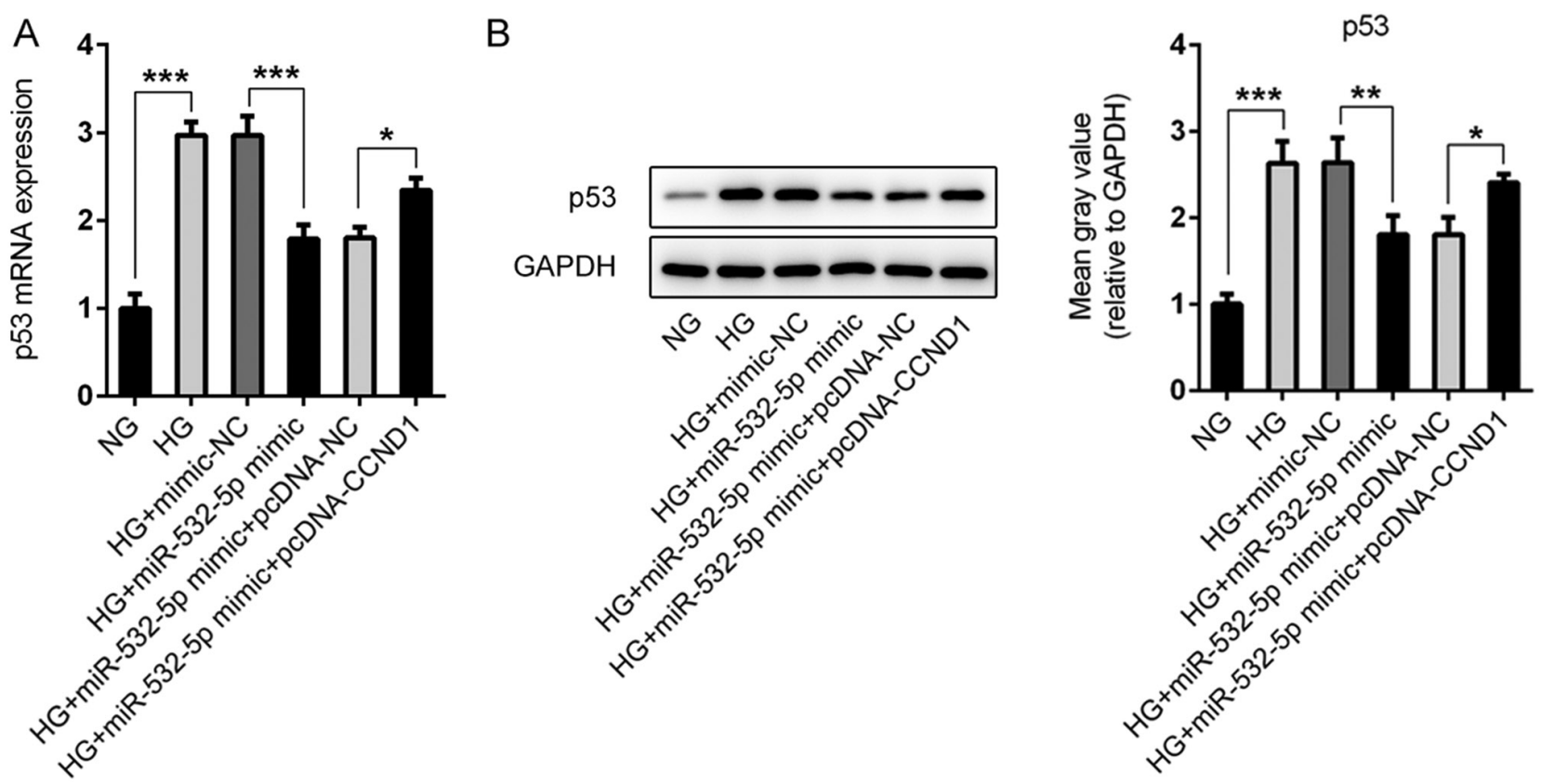

Figure 8. Overexpression of miR-532-5p regulates the expression of p53 in HG-induced cells by downregulating CCND1. p53 expression was determined using (A) reverse transcription-quantitative PCR and (B) western blotting. ${ }^{*} \mathrm{P}<0.05,{ }^{* * *} \mathrm{P}<0.01,{ }^{* * * *} \mathrm{P}<0.001$. HG, high glucose; miR, microRNA; MA, mannitol group; NG, normal glucose; NC, negative control; CCND1, cyclin D1.

At present, studies on miR-532-5p are concerned with its role in other diseases. miR-532-5p expression is downregulated in $\mathrm{H} 9 \mathrm{C} 2$ cells exposed to hypoxia, and in the myocardium of rats with acute myocardial infarction, to reduce the apoptosis of $\mathrm{H} 9 \mathrm{C} 2$ cells (8). In addition, miR-532-5p expression is downregulated in LPS-induced inflammation of RAW264.7 macrophages (23). It has been revealed that miR-532-5p serves an important role in the inflammatory response (24), oxidative stress (25) and apoptosis (8). However, to the best of our knowledge, the role of miR-532-5p in diabetes has not yet been reported. In the present study, it was revealed that overexpression of miR-532-5p could increase the secretion of insulin in HG-induced cells. Furthermore, miR-532-5p could inhibit the levels of oxidative stress in cells, and could inhibit the apoptosis of HG-induced cells.

The targeted binding of miR-532-5p to CCND1 was identified using the StarBase website, and this targeting relationship was further verified in the present study using a luciferase reporter assay. Studies have reported that the expression of CCND1 in diabetes is upregulated $(9,26)$. However, to the best of our knowledge, the specific role of CCND1 in diabetes has not yet been reported. In the present study, it was revealed that the expression levels of CCND1 were upregulated in HG-induced cells, and overexpression of miR-532-5p inhibited CCND1 expression, suggesting that miR-532-5p targeting CCND1 served a regulatory role in diabetes. It has been reported that inhibition of CCND1 expression can block the cell cycle and inhibit cell proliferation (27). Furthermore, in multiple myeloma, CCND1 can control redox metabolism by producing ROS to disrupt the redox balance (11). In the present study, upregulation of miR-532-5p was proposed to regulate insulin secretion, oxidative stress and apoptosis in HG-induced cells by downregulating CCND1 expression. The experimental step of knocking down the expression of CCND1 will be further explored in our future research.

During the experiment, it was revealed that overexpression of miR-532-5p regulated the expression levels of p53 in HG-induced MIN6 cells by downregulating CCND1 expression. A previous study reported that adaptive EGF expression sensitized pancreatic cancer cells to ionizing radiation by activating the CCND1/p53/PARP signaling pathway (15). Following glucose treatment, the expression levels of p53 increase in a concentration-dependent manner, and the upregulation of $\mathrm{p} 53$ induces apoptosis and reduces insulin secretion (14). Therefore, it was preliminarily concluded that miR-532-5p regulated oxidative stress and insulin secretion of pancreatic $\beta$ cells induced by HG by downregulating CCND1 expression, which may be regulated via the modulation of p53 expression levels. Further experimental verification of this preliminary conclusion will be carried out in subsequent research.

Due to the length of the article, only in vitro experiments were performed and described in the present study. In addition, our research group is conducting the relevant in vivo experiments to further verify the conclusion obtained from these in vitro experiments.

In conclusion, the present study revealed that miR-532-5p regulated oxidative stress and insulin secretion in HG-induced pancreatic $\beta$ cells by downregulating the expression levels of CCND1, which was involved in the regulation of p53 expression.

\section{Acknowledgements}

Not applicable.

\section{Funding}

No funding was received. 


\section{Availability of data and materials}

The datasets used and/or analyzed during the current study are available from the corresponding author on reasonable request.

\section{Authors' contributions}

$\mathrm{ZZ}$ and WS made substantial contributions to the conception and design of the study, and the acquisition of data. HC made substantial contributions to analysis and interpretation of data. $\mathrm{ZZ}$ and WS confirm the authenticity of all the raw data. All authors read and approved the final manuscript.

\section{Ethics approval and consent to participate}

Not applicable.

\section{Patients consent for publication}

Not applicable.

\section{Competing interests}

The authors declare that they have no competing interests.

\section{References}

1. Yang X, Zhang Y, Xu W, Deng R, Liu Y, Li F, Wang Y, Ji X, Bai M, Zhou F, et al: Potential role of Hsp90 in rat islet function under the condition of high glucose. Acta Diabetol 53: 621-628, 2016.

2. Zhao YC, Zhu J, Song GY and Li XS: Relationship between thioredoxin-interacting protein (TXNIP) and islet $\beta$-cell dysfunction in patients with impaired glucose tolerance and hypertriglyceridemia. Int J Clin Exp Med 8: 4363-4368, 2015.

3. Nelson P, Smith N, Ciupe S, Zou W, Omenn GS and Pietropaolo M: Modeling dynamic changes in type 1 diabetes progression: Quantifying beta-cell variation after the appearance of islet-specific autoimmune responses. Math Biosci Eng 6: 753-778, 2009.

4. Dahan T, Ziv O, Horwitz E, Zemmour H, Lavi J, Swisa A, Leibowitz G, Ashcroft FM, In't Veld P, Glaser B and Dor Y: Pancreatic beta-cells express the fetal islet hormone gastrin in rodent and human diabetes. Diabetes 66: 426-436, 2017.

5. Saliminejad K, Khorram Khorshid HR, Soleymani Fard S and Ghaffari SH: An overview of microRNAs: Biology, functions, therapeutics, and analysis methods. J Cell Physiol 234: $5451-5465,2019$

6. Jones A, Danielson KM, Benton MC, Ziegler O, Shah R, Stubbs RS, Das S and Macartney-Coxson D: MiRNA signatures of insulin resistance in obesity. Obesity (Silver Spring) 25 1734-1744, 2017.

7. Ortega FJ, Mercader JM, Moreno-Navarrete JM, Rovira O, Guerra E, Esteve E, Xifra G, Martínez C, Ricart W, Rieusset J, et al: Profiling of circulating microRNAs reveals common microRNAs linked to type 2 diabetes that change with insulin sensitization. Diabetes Care 37: 1375-1383, 2014.

8. Ma J, Zhang J, Wang Y, Long K, Wang X, Jin L, Tang Q, Zhu L, Tang G, Li X and Li M: MiR-532-5p alleviates hypoxia-induced cardiomyocyte apoptosis by targeting PDCD4. Gene 675: 36-43, 2018.

9. Taneera J, Fadista J, Ahlqvist E, Zhang M, Wierup N, Renström E and Groop L: Expression profiling of cell cycle genes in human pancreatic islets with and without type 2 diabetes. Mol Cell Endocrinol 375: 35-42, 2013.

10. Shen J and Zhu B: Integrated analysis of the gene expression profile and DNA methylation profile of obese patients with type 2 diabetes. Mol Med Rep 17: 7636-7644, 2018.
11. Bustany S, Bourgeais J, Tchakarska G, Body S, Hérault O, Gouilleux F and Sola B: Cyclin D1 unbalances the redox status controlling cell adhesion, migration, and drug resistance in myeloma cells. Oncotarget 7: 45214-45224, 2016

12. Zhong Q, Hu Z, Li Q, Yi T, Li J and Yang H: Cyclin D1 silencing impairs DNA double strand break repair, sensitizes BRCA1 wildtype ovarian cancer cells to olaparib. Gynecol Oncol 152: $157-165,2019$.

13. Pistritto G, Trisciuoglio D, Ceci C, Garufi A and D'Orazi G: Apoptosis as anticancer mechanism: Function and dysfunction of its modulators and targeted therapeutic strategies. Aging (Albany NY) 8: 603-619, 2016.

14. Liu RX, Ma Y, Hu XL, Liao YP, Hu XN, He BC and Sun WJ: Pioglitazone/metformin adduct regulates insulin secretion and inhibits high glucose-induced apoptosis via p21-p53-MDM2 signaling in INS-1 cells. J Cell Biochem 119: 5449-5459, 2018.

15. Liu X, Chen H, Hou Y, Ma X, Ye M, Huang R, Hu B, Cao H, Xu L, Liu M, et al: Adaptive EGF expression sensitizes pancreatic cancer cells to ionizing radiation through activation of the cyclin D1/P53/PARP pathway. Int J Oncol 54: 1466-1480, 2019.

16. Ruan D, Liu Y, Wang X, Yang D and Sun Y: MiR-149-5p protects against high glucose-induced pancreatic beta cell apoptosis via targeting the $\mathrm{BH} 3$-only protein BIM. Exp Mol Pathol 110: $104279,2019$.

17. Livak KJ and Schmittgen TD: Analysis of relative gene expression data using real-time quantitative PCR and the 2(-Delta Delta C(T)) method. Methods 25: 402-408, 2001.

18. Abouzaripour M, Pasbakhsh P, Atlasi N, Shahverdi AH, Mahmoudi R and Kashani IR: In vitro differentiation of insulin secreting cells from mouse bone marrow derived stage-specific embryonic antigen 1 positive stem cells. Cell J 17: 701-710, 2016.

19. Ye D, Zhang T, Lou G, Xu W, Dong F, Chen G and Liu Y: Plasma miR-17, miR-20a, miR-20b and miR-122 as potential biomarkers for diagnosis of NAFLD in type 2 diabetes mellitus patients. Life Sci 208: 201-207, 2018.

20. Lakhter AJ, Pratt RE, Moore RE, Doucette KK, Maier BF, DiMeglio LA and Sims EK: Beta cell extracellular vesicle miR-21-5p cargo is increased in response to inflammatory cytokines and serves as a biomarker of type 1 diabetes. Diabetologia 61: 1124-1134, 2018.

21. Tang Q, Len Q, Liu Z and Wang W: Overexpression of miR-22 attenuates oxidative stress injury in diabetic cardiomyopathy via Sirt 1. Cardiovasc Ther 36, 2018.

22. Wang J, Zhang J, Chen X, Yang Y, Wang F, Li W, Awuti M, Sun Y, Lian C, Li Z, et al: MiR-365 promotes diabetic retinopathy through inhibiting Timp3 and increasing oxidative stress. Exp Eye Res 168: 89-99, 2018

23. Cheng Y, Kuang W, Hao Y, Zhang D, Lei M, Du L, Jiao H, Zhang $X$ and Wang F: Downregulation of miR-27a* and miR-532-5p and upregulation of miR-146a and miR-155 in LPS-induced RAW264.7 macrophage cells. Inflammation 35: 1308-1313, 2012

24. Yan X, Zeng D, Zhu H, Zhang Y, Shi Y, Wu Y, Tang $\mathrm{H}$ and Li D: MiRNA-532-5p regulates CUMS-induced depression-like behaviors and modulates LPS-induced proinflammatory cytokine signaling by targeting STAT3. Neuropsychiatr Dis Treat 16: 2753-2764, 2020

25. Cai X, Wang S, Hong L, Yu S, Li B, Zeng H, Yang X, Zhang P and Shao L: Long noncoding RNA taurine-upregulated gene 1 knockdown protects cardiomyocytes against hypoxia/reoxygenation-induced injury through regulating $\mathrm{miR}-532-5 \mathrm{p} / \mathrm{Sox} 8$ axis. J Cardiovasc Pharmacol 76: 556-563, 2020.

26. Gurke J, Schindler M, Pendzialek SM, Thieme R, Grybel KJ, Heller R, Spengler K, Fleming TP, Fischer B and Navarrete Santos A: Maternal diabetes promotes mTORC1 downstream signalling in rabbit preimplantation embryos. Reproduction 151: 465-476, 2016.

27. Li N, Zeng J, Sun F, Tong X, Meng G, Wu C, Ding X, Liu L, Han M, Lu C and Dai F: p27 inhibits CDK6/CCND1 complex formation resulting in cell cycle arrest and inhibition of cell proliferation. Cell Cycle 17: 2335-2348, 2018.

This work is licensed under a Creative Commons Attribution-NonCommercial-NoDerivatives 4.0 International (CC BY-NC-ND 4.0) License. 